\title{
Exponential operators for Fredholm singular integral equations and space - fractional PDEs
}

\author{
Arman Aghili \\ Department of Applied Mathematics, Faculty of Mathematical Sciences, University of Guilan, Rasht, Iran
}

Received: 30 January 2016, Accepted: 19 April 2016

Published online: 16 October 2016.

\begin{abstract}
In this article, it is shown that the combined use of exponential operators and special functions provides a powerful tool to solve certain type of fractional PDEs. A system of space fractional partial differential equations is solved. The exponential operators are powerful and effective method for solving certain singular integral equations and fractional partial differential equations with nonconstant coefficients. Constructive examples are also provided.
\end{abstract}

Keywords: Fractional partial differential equations, Riemann Liouville fractional derivative, Fredholm singular integral equation, Laplace transforms.

\section{Introduction and Preliminaries}

We present a general method of operational nature to obtain solutions for several types of partial differential equations.

Definition 1. Laplace transform of function $f(t)$ is as follows

$$
L\{f(t)\}=\int_{0}^{\infty} e^{-s t} f(t) d t:=F(s)
$$

If $L\{f(t)\}=F(s)$, then $L^{-1}\{F(s)\}$ is given by

$$
f(t)=\frac{1}{2 \pi i} \int_{c-i \infty}^{c+i \infty} e^{s t} F(s) d s
$$

where $F(s)$ is analytic in the region $\mathrm{R} e(s)>c$.

Definition 2. If the function $\Phi(t)$ belongs to $C[a, b]$ and $a<t<b$,The left Riemann-Liouville fractional integral of order $\alpha>0$ is defined as

$$
I_{a}^{R L, \alpha}\{\Phi(t)\}=\frac{1}{\Gamma(\alpha)} \int_{a}^{t} \frac{\Phi(\xi)}{(t-\xi)^{1-\alpha}} d \xi
$$

The left Riemann-Liouville fractional derivative of order $\alpha>0$ is defined as

$$
D_{a}^{R L, \alpha} \phi(x)=\frac{1}{\Gamma(1-\alpha)} \frac{d}{d x} \int_{a}^{t} \frac{\Phi(\xi)}{(t-\xi)^{\alpha}} d \xi
$$

It follows that $D_{a}^{R L, \alpha} \phi(x)$ exists for all $\Phi(t)$ belongs to $C[a, b]$, and $a<t<b$. 
Note: A very useful fact about the R-L operators is that they satisfy semi group properties of fractional integrals. The special case of fractional derivative when $\alpha=0.5$ is called semi derivative.

Definition 3. The left Caputo fractional derivative of order $\alpha(0<\alpha<1)$ of $\phi(t)$ is as follows

$$
D_{a}^{c, \alpha} \phi(x)=\frac{1}{\Gamma(1-\alpha)} \int_{a}^{t} \frac{1}{(t-\xi)^{\alpha}} \phi^{\prime}(\xi) d \xi
$$

Lemma 1. Let $L\{f(t)\}=F(s)$, then, the following identities hold true.

(1) $\left.L^{-1} F\left(s^{\beta}\right)=\frac{1}{2 \pi} \int_{0}^{\infty} f(\tau)\left(\int_{0}^{\infty} e^{-t r-r^{\beta}(\tau \cos \beta \pi)} \sin \left(r^{\beta} \tau \sin \beta \pi\right)\right) d r\right) d \tau$;

(2) $L^{-1} F(\sqrt{s})=\frac{1}{(2 t \sqrt{t \pi})} \int_{0}^{\infty} e^{\frac{\xi^{2}}{-4 t}} f(\xi) d \xi$;

(3) $L^{-1}\left(e^{-k \sqrt{s}}\right)=\frac{k}{(2 \sqrt{\pi})} \int_{0}^{\infty} e^{-s \xi-\frac{k^{2}}{4 \xi}} d \xi$;

(4) $e^{-\omega s^{\beta}}=\frac{1}{\pi} \int_{0}^{\infty} e^{-r^{\beta}(\omega \cos \beta \pi)} \sin \left(\omega r^{\beta} \sin \beta \pi\right)\left(\int_{0}^{\infty} e^{-s \tau-r \tau} d \tau\right) d r$;

Proof. See [1].

Lemma 2. The following exponential identities hold true.

(1) $\exp \left( \pm \lambda \frac{d}{d t}\right) \Phi(t)=\Phi(t \pm \lambda)$;

(2) $\exp \left( \pm \lambda t \frac{d}{d t}\right) \Phi(t)=\Phi\left(t e^{ \pm \lambda}\right)$;

(3) $\exp \left(\lambda \sqrt{t} \frac{d}{d t}\right) \Phi(t)=\Phi\left(\left(\sqrt{t}+\frac{\lambda}{2}\right)^{2}\right)$;

(4) $\exp \left(\lambda q(t) \frac{d}{d t}\right) \Phi(t)=\Phi(Q(F(t)+\lambda))$;

where $F(t)$ is primitive of $\frac{1}{q(t)}$ and $Q(t)$ is inverse of $F(t)$.

Proof. See [4].

The Laplace transform is useful tool in applied mathematics, for instance for solving singular integral equations, partial differential equations, and in automatic control,where it defines a transfer function.

Example 1. Let us consider the following non linear impulsive differential equation

$$
\left(\sqrt{D_{t}-a}\right) y(t)=\delta(x-\lambda)
$$

Solution can be found using of part 3 of lemma (1), the above differential equation can be written as below

$$
y(t)=\frac{1}{\left(\sqrt{D_{t}-a}\right)} \delta(x-\lambda)
$$

from which we deduce

$$
y(t)=\int_{0}^{\infty} d \xi \frac{e^{-a \xi}}{\sqrt{a+\xi}} e^{-\xi D_{t}} \delta(t-\lambda)
$$

finally,using elementary properties of Dirac delta function leads to the following solution

$$
y(t)=\int_{0}^{\infty} d \xi \frac{e^{-a \xi}}{\sqrt{a+\xi}} \delta(t-\xi-\lambda)=\frac{e^{-a(t-\lambda)}}{\sqrt{\pi(a+t-\lambda)}},
$$

Example 2. Show that the following exponential identities hold true.

(1) $\exp \left(-\frac{k^{2}}{2 t} \frac{d}{d t}\right) \Phi(t)=\Phi\left(\sqrt{\left.t^{2}-k^{2}\right)}\right.$;

(2) $\exp \left(-k t^{2} \frac{d}{d t}\right) \Phi(t)=\Phi\left(\frac{t}{k+t}\right)$; 
Let us take $-\frac{k^{2}}{2}=\lambda$ and $q(t)=\frac{1}{t}$ then we get $\frac{1}{q(t)}=t$ and $F(t)=\frac{t^{2}}{2}$ where $F(t)$ is primitive of $\frac{1}{q(t)}$. $Q(t)$ inverse of $F(t)$ is $Q(t)=\sqrt{2 t}$. Now, direct application of part 4 of lemma 2 leads to the following

$$
\exp \left(-\frac{k^{2}}{2 t} \frac{d}{d t}\right) \Phi(t)=\Phi\left(\sqrt{\left.t^{2}+2 \lambda\right)}=\Phi\left(\sqrt{t^{2}-k^{2}}\right)\right.
$$

Let us take $-t^{2}=q(t)$ then $\frac{1}{q(t)}=-\frac{1}{t^{2}}$ from which we get $F(t)=\frac{1}{t}$ where $F(t)$ is primitive of $\frac{1}{q(t)}$. $Q(t)$ inverse of $F(t)$, therefore, we get $Q(t)=\frac{1}{t}$.In view of part 4 of lemma 1.2, one has

$$
\exp \left(-k t^{2} \frac{d}{d t}\right) \Phi(t)=\Phi\left(\frac{t}{k+t}\right) .
$$

Example 3. Let us solve the following fractional Volterra equation of convolution type. The Laplace transform provides a useful technique for the solution of such fractional singular integro- differential equations.

$$
\lambda \int_{0}^{t} \cosh (a(t-\xi)) D^{\alpha} \phi(\xi) d \xi=\left(\frac{t}{a}\right)^{\frac{\mu}{2}} I_{\mu}(2 \sqrt{a t}) \quad \phi(0)=0,
$$

Solution. Upon taking the Laplace transform of the given integral equation, we obtain

$$
s^{\alpha} \Phi(s) \frac{s \lambda}{\left(s^{2}-a^{2}\right)}=\frac{e^{\frac{a}{s}}}{s^{1+\mu}},
$$

solving the above equation, leads to

$$
\Phi(s)=\frac{\left(s^{2}-a^{2}\right) e^{\frac{a}{s}}}{(a \lambda) s^{2+\alpha+\mu}},
$$

or equivalently

$$
\Phi(s)=\frac{\left(s^{2} e^{\frac{a}{s}}-a^{2} e^{\frac{a}{s}}\right)}{(a \lambda) s^{2+\alpha+\mu}},
$$

at this point, taking inverse Laplace transform term wise, after simplifing we obtain

$$
\phi(t)=\frac{1}{a \lambda}\left(\frac{t}{a}\right)^{\frac{\alpha+v}{2}} I_{\alpha+\mu}(2 \sqrt{a t})-\frac{a}{\lambda}\left(\frac{t}{a}\right)^{\frac{(\alpha+\mu+2)}{2}} I_{\alpha+v+2}(2 \sqrt{a t}),
$$

Note: $I_{\eta}($.$) , stands for modified Bessel's function of first kind of order \eta$.

Corollary 1. Let us consider the following singular integral equation

$$
\exp \left(-\eta x^{m}\right)=\int_{0}^{\infty} \xi^{v} e^{-\xi} g\left(x \xi^{\mu}\right) d \xi
$$

the above integral equation has the following formal solution

$$
g(x)=J_{v}(\sqrt[m]{\eta} x: m \mu)
$$

Where $J_{v}($. . .) stands for the Bessel-Wright function of order $v$.

Note:The special function of the form defined by the series representation

$$
J_{v}(x: \mu)=\sum_{n=0}^{\infty} \frac{(-1)^{n}}{n ! \Gamma(1+v+n \mu)}(x)^{n}
$$

is known as Bessel-Maitland function or the Bessel-Wright function.It has a wide application in the problem of physics, chemistry, applied sciences. 
Proof. Let us rewrite the right hand side of the above equation(7) as below

$$
\exp \left(-\eta x^{m}\right)=\left(\int_{0}^{\infty} d \xi \xi^{v} e^{-\xi^{\mu} D_{x}} g(x)\right)
$$

in relation (8), we used the following exponential identity

$$
\Phi\left(\lambda^{k} x\right)=\Phi\left(e^{k \ln (\lambda)} x\right)=\Phi\left(e^{\ln (\lambda)^{k}} x\right)=e^{\ln (\lambda)^{k} x D_{x}} \Phi(x)=\lambda^{k x D_{x}} \Phi(x),
$$

thus, it follows that

$$
\exp \left(-\eta x^{m}\right)=\left(\int_{0}^{\infty} \xi^{v} e^{-\xi^{\mu} D_{x}} d \xi\right) g(x)
$$

At this point, we may rewrite relation (9) in terms of Gamma function as follows

$$
\exp \left(-\eta x^{m}\right)=\left(\int_{0}^{\infty} \xi^{v} e^{-\xi^{\mu} D_{x}} d \xi\right) g(x)=\Gamma\left(1+v+x \mu D_{x}\right) g(x)
$$

From the above operational relationship and Taylor expansion of the exponential function results in

$$
\begin{aligned}
& g(x)=\sum_{n=0}^{\infty} \frac{(-1)^{n}}{\Gamma\left(1+v+\mu x D_{x}\right)}\left((\eta)^{\frac{1}{m}} x\right)^{n m} . \\
& g(x)=\sum_{n=0}^{\infty} \frac{(-1)^{n}}{\Gamma(1+v+m n \mu)}(\sqrt[m]{\eta} x)^{m n}=J_{v}(\sqrt[m]{\eta} x: m \mu) .
\end{aligned}
$$

Corollary 2. Let us consider the following singular integral equation

$$
x^{v} \exp (x)=\left(\int_{0}^{\infty} \xi^{v} e^{-\xi} g(x \xi) d \xi\right)
$$

the above integral equation has the following formal solution

$$
g(x)=I_{2 v}(2 \sqrt{x})
$$

where $I_{2 v}(2 \sqrt{x})$, stands for the modified Bessel function of first kind of order $v$.

Proof. Let us rewrite the left hand side of the above equation as below

$$
x^{v} \exp (x)=\left(\int_{0}^{\infty} d \xi e^{-\xi} \xi^{x D_{x}+v}\right) g(x),
$$

and treating the derivative operator as a constant, the evaluation of the integral yields

$$
g(x)=\Gamma^{-1}\left(1+v+x D_{x}\right)\left(x^{v} \exp (x)\right)
$$

after writing Taylor expansion of exponential function, one gets

$$
g(x)=\sum_{n=0}^{\infty} \frac{(1)}{n ! \Gamma\left(1+v+x D_{x}\right)}(x)^{n+v}=\sum_{n=0}^{\infty} \frac{(1)}{n ! \Gamma(1+2 v+n)}(x)^{n+v}=I_{2 v}(2 \sqrt{x}) .
$$


Note: From operational relation $\left(x D_{x}\right) x^{n}=n x^{n}$ we get the following identity

$$
g\left(c x D_{x}\right) x^{n}=g(c n) x^{n} .
$$

and $g(x)$ has Taylor series expansion.

Lemma 3. The following exponential operator identity holds true

$$
\left(\frac{d}{d t}\right)^{\left(-\frac{v}{m}\right)} \exp \left(\lambda \frac{d}{d t}\right) \Phi(t)=\frac{1}{\Gamma\left(\frac{v}{m}\right)} \int_{0}^{\infty} \frac{\Phi(t+\lambda-\xi)}{\sqrt[m]{(\xi)^{m-v}}} d \xi .
$$

Proof. Let us introduce the following integral

$$
I(p)=\frac{1}{\Gamma\left(\frac{v}{m}\right)} \int_{-\lambda}^{\infty} \frac{\exp (-p \xi)}{\sqrt[m]{(\lambda+\xi)^{m-v}}} d \xi
$$

By making the change of variable $\lambda+\xi=\zeta$ in the above integral we get

$$
I(p)=\frac{1}{\Gamma\left(\frac{v}{m}\right)} \int_{0}^{\infty} \frac{\exp (p \lambda-p \zeta)}{\sqrt[m]{(\zeta)^{m-v}}} d \zeta
$$

after simplifying,one gets

$$
I(p)=\frac{\exp (\lambda p)}{\Gamma\left(\frac{v}{m}\right)} \int_{0}^{\infty} \frac{\exp (-p \zeta)}{\sqrt[m]{(\zeta)^{m-v}}} d \zeta
$$

or,

$$
I(p)=\frac{\exp (\lambda p)}{\sqrt[m]{(p)^{v}}}
$$

Let us choose $p=\frac{d}{d t}$, we obtain

$$
\left(\frac{d}{d t}\right)^{\left(-\frac{v}{m}\right)} \exp \left(\lambda \frac{d}{d t}\right) \Phi(t)=\frac{1}{\Gamma\left(\frac{v}{m}\right)} \int_{0}^{\infty} \frac{\Phi(t+\lambda-\xi)}{\sqrt[m]{(\xi)^{m-v}}} d \xi .
$$

Special case. For $m=3, v=2$, we obtain

$$
\left(\frac{d}{d t}\right)^{-\frac{2}{3}} \exp \left(\lambda \frac{d}{d t}\right) \Phi(t)=\frac{1}{\Gamma\left(\frac{2}{3}\right)} \int_{0}^{\infty} \frac{\Phi(t+\lambda-\xi)}{\sqrt[3]{\xi}} d \xi .
$$

Lemma 4. The following second order exponential operator relations hold true.

(1) $\exp \left(r\left(\frac{\partial}{\partial x}\right)^{2}\right) \Phi(x)=\frac{1}{(2 r \sqrt{\pi})} \int_{0}^{\infty} e^{-\frac{u^{2}}{4 r}}(\Phi(x+i u)+\Phi(x-i u)) d u$

(2) $\exp \left(k x\left(\frac{\partial}{\partial x}\right)^{2}\right) \Phi(x)=\frac{1}{(2 k x \sqrt{\pi})} \int_{0}^{\infty} e^{-\frac{u^{2}}{4 k x}}(\Phi(x+i u)+\Phi(x-i u)) d u$.

Proof. Let us consider the following elementary integral

$$
r \sqrt{\pi} \exp \left(-\left(b^{2}-a^{2}\right) r\right)=\int_{0}^{\infty} e^{-\frac{u^{2}}{4 r}} \cos (a u) \cosh (b u) d u .
$$

By integration by parts one can easily find the value of the integral and after some manipulations we obtain

$$
\exp \left(-\left(b^{2}-a^{2}\right) r\right)=\frac{1}{(4 r \sqrt{\pi})} \int_{0}^{\infty} e^{-\frac{u^{2}}{4 r}}(\exp (i a u)+\exp (-i a u))(\exp (b u)+\exp (-b u)) d u
$$


(1) In the above integral relation, we set $a=\left(\frac{\partial}{\partial x}\right), b=0$ to obtain

$$
\exp \left(r\left(\frac{\partial}{\partial x}\right)^{2}\right) \Phi(x)=\frac{1}{(2 r \sqrt{\pi})} \int_{0}^{\infty} d u\left(e^{-\frac{u^{2}}{4 r}}\left(\exp (i u)\left(\frac{\partial}{\partial x}\right)+\exp (-i u)\left(\frac{\partial}{\partial x}\right)\right) \Phi(x)\right.
$$

by using lemma 1 , we get finally

$$
\exp \left(r\left(\frac{\partial}{\partial x}\right)^{2}\right) \Phi(x)=\frac{1}{(2 r \sqrt{\pi})} \int_{0}^{\infty} e^{-\frac{u^{2}}{4 r}}(\Phi(x+i u)+\Phi(x-i u)) d u
$$

(2) In the above integral relation, we set $r=k x a=\frac{\partial}{\partial x}, b=0$ to obtain

$$
\exp \left(k x\left(\frac{\partial}{\partial x}\right)^{2}\right) \Phi(x)=\frac{1}{(2 k x \sqrt{\pi})} \int_{0}^{\infty} d u\left(e^{-\frac{u^{2}}{4 k x}}\left(\exp (i u)\left(\frac{\partial}{\partial x}\right)+\exp (-i u)\left(\frac{\partial}{\partial x}\right)\right) \Phi(x)\right.
$$

by using lemma 1 , we arrive at

$$
\exp \left(k x\left(\frac{\partial}{\partial x}\right)^{2}\right) \Phi(x)=\frac{1}{(2 k x \sqrt{\pi})} \int_{0}^{\infty} e^{-\frac{u^{2}}{4 k x}}(\Phi(x+i u)+\Phi(x-i u)) d u
$$

Corollary 3. Let us consider the following Fredholm singular integral equation

$$
\exp \left(-\eta x^{2}\right)=\int_{-\infty}^{\infty} e^{-\xi^{2}} \phi(x+2 \xi \sqrt{\lambda}) d \xi
$$

the above integral equation has the following formal solution

$$
\Phi(x)=\frac{\exp \left(\frac{-\lambda x^{2}(5-4 \lambda \eta)}{1-4 \lambda \eta}\right)}{\sqrt{\pi \lambda(1-4 \lambda \pi)}}
$$

Proof. Let us rewrite the right hand side of the above equation as below

$$
\exp \left(-\eta x^{2}\right)=\int_{-\infty}^{\infty} d \xi e^{-\xi^{2}} e^{-2 \sqrt{\lambda} \xi D_{x}} \Phi(x)
$$

and treating the derivative operator as a constant, the evaluation of the integral yields

$$
\Phi(x)=\frac{1}{\sqrt{\pi}} e^{-\lambda D_{x}^{2}} \exp \left(-\eta x^{2}\right)
$$

at this point, using relation (22), one has

$$
\Phi(x)=\frac{1}{\sqrt{\pi}} \frac{1}{(2 \lambda \sqrt{\pi})} \int_{0}^{\infty} e^{-\frac{u^{2}}{4 \lambda}}\left(\exp \left(-\eta(x+i u)^{2}\right)+\exp \left(-\eta(x-i u)^{2}\right)\right) d u
$$

or,

$$
\left.\Phi(x)=\frac{\exp \left(-\lambda x^{2}\right)}{(2 \lambda \pi}\right) \int_{0}^{\infty} \exp \left(-\left(\left(\frac{1}{4 \lambda}\right)-\eta\right) u^{2}\right) \cos 2 x u d u
$$


from which and after some easy calculations, we get

$$
\Phi(x)=\frac{\exp \left(\frac{-\lambda x^{2}(5-4 \lambda \eta)}{1-4 \lambda \eta}\right)}{\sqrt{\pi \lambda(1-4 \lambda \pi)}},
$$

\section{Evaluation of Certain Integrals}

The main purpose of this section is to introduce the use of exponential differential operator technique for evaluation of certain types of integrals. Where $E_{\alpha, \beta}(. .$.$) stands for the Mittag-Leffler function with parameters \alpha, \beta$.

Note:The special function of the form defined by the following series representation

$$
E_{\alpha, \beta}(x)=\sum_{n=0}^{\infty} \frac{(1)}{n ! \Gamma(\beta+n \alpha)}(x)^{n}
$$

is known as Mittag - Leffler function with two papameters.It has a wide application in the problem of physics, chemistry,engineering, applied mathematical sciences.

Lemma 5. Considering the integral

$$
I_{0}=I(x, \alpha, \beta)=\int_{0}^{\infty} E_{\alpha, \beta}\left(\frac{x}{\left(k^{2}+t^{2}\right)^{\mu}}\right) d t
$$

as a function with parameters $\alpha, \beta$, show thatI $(x, \alpha, \beta)$ satisfies the following relationship

$$
I_{0}=\int_{0}^{\infty} E_{\alpha, \beta}\left(\frac{x}{\left(k^{2}+t^{2}\right)^{\mu}}\right) d t=\frac{k \sqrt{\pi}}{2} \sum_{n=0}^{\infty} \frac{1}{n ! \Gamma(\beta+n \alpha)} \frac{\Gamma(\mu(n+v)-0.5)}{\Gamma(\mu(n+v))}\left(k^{-2 \mu} x\right)^{n},
$$

Proof. By making a change of variable $t=k y$, and letting $x=k^{2 \mu} r$, we get

$$
I_{0}=k \int_{0}^{\infty} E_{\alpha, \beta}\left(\frac{r}{\left(1+y^{2}\right)^{\mu}}\right) d y
$$

The above integral can be written in the following operational form

$$
I_{0}=k \int_{0}^{\infty} E_{\alpha, \beta}\left(\frac{r}{\left(1+y^{2}\right)^{\mu}}\right) d y=k\left(\int_{0}^{\infty}\left(\frac{1}{1+y^{2}}\right)^{\mu r D_{r}} d y\right) E_{\alpha, \beta}(r),
$$

after evaluation and simplifying the right hand side integral, this last result leads to

$$
I_{0}=k \int_{0}^{\infty} E_{\alpha, \beta}\left(\frac{r}{\left(1+y^{2}\right)^{\mu}}\right) d y=\frac{k \sqrt{\pi}}{2} \frac{\Gamma\left(\mu r D_{r}-0.5\right)}{\Gamma\left(\mu r D_{r}\right)} E_{\alpha, \beta}(r) .
$$

By using Taylor expansion of the Mittag-Leffler function with parameters $\alpha, \beta$, one has

$$
I_{0}=\frac{k \sqrt{\pi}}{2} \sum_{n=0}^{\infty} \frac{1}{n ! \Gamma(\beta+n \alpha)} \frac{\Gamma\left(\mu r D_{r}-0.5\right)}{\Gamma\left(\mu r D_{r}\right)}(r)^{n},
$$

finally,

$$
I_{0}=\frac{k \sqrt{\pi}}{2} \sum_{n=0}^{\infty} \frac{1}{n ! \Gamma(\beta+n \alpha)} \frac{\Gamma(\mu(n+v)-0.5)}{\Gamma(\mu(n+v))}\left(k^{-2 \mu} x\right)^{n}
$$




\section{Fokker-Planck equation}

The classical Fokker-Planck equation is a linear parabolic equation which describes the time evolution of probability distribution of a stochastic process defined on a Euclidean space. The Fokker-Planck equation provides a very useful tool for modelling a wide variety of stochastic phenomena arising in physics,,chemistry, biology, finance, traffic flow, etc. Given the importance of the Fokker-Planck equation, different analytical and numerical methods have been proposed for its solution.As it is well known the stationary solution of Fokker-Planck equation can be given in a closed form if the condition of a detailed balance holds. The study of the time-dependent solution is a much more complicated problem. In this section,the space fractional Fokker-Planck equation (42) with a general time-dependence and a special x-dependence of the drift and diffusion coefficients has been studied analytically.

Lemma 6. Let us consider the following space-fractional Fokker-Planck equation

$$
\begin{aligned}
& u_{t}+\alpha\left(\sqrt{\frac{\partial}{\partial x} x \frac{\partial}{\partial x}}\right) u+\lambda x^{v} u(x)=0, \\
& u(x, 0)=f(x) .
\end{aligned}
$$

Proof. In order to obtain a solution for equation (42)-(43) in view of [2],[3] first by solving the first order PDE with respect to $t$, and applying the initial condition (43), we get the following relationship

$$
u(x, t)=\exp \left(-\lambda t x^{v}\right) \exp \left(-a t\left(\frac{\partial}{\partial x} x \frac{\partial}{\partial x}\right)^{0.5}\right) f(x)
$$

In order to find the result of the action of exponential operator, we make use of lemma 1 , by choosing $\omega=a t, s=\left(\frac{\partial}{\partial x} x \frac{\partial}{\partial x}\right)$, to obtain

$$
u(x, t)=\exp \left(-\lambda t x^{v}\right) \exp \left(-a t\left(\frac{\partial}{\partial x} x \frac{\partial}{\partial x}\right)^{0.5}\right) f(x)
$$

or

(i) $u(x, t)=\exp \left(-\lambda t x^{v}\right) \frac{1}{\pi} \int_{0}^{\infty} e^{-r^{\lambda}(a t \cos 0.5 \pi)} \sin \left(a t r^{0.5} \sin 0.5 \pi\right)\left(\int_{0}^{\infty} d \tau e^{-r \tau-\tau \frac{\partial}{\partial x} x \frac{\partial}{\partial x}} f(x)\right) d r$;

(ii) Let us take $f(x)=\exp (-q x)$,

$$
u(x, t)=\exp \left(-\lambda t x^{\mu}\right) \frac{1}{\pi} \int_{0}^{\infty} e^{-r^{0.5}\left(a t^{v+1} \cos 0.5 \pi\right)} \sin \left(a t r^{0.5} \sin 0.5 \pi\right)\left(\int_{0}^{\infty} d \tau e^{-r \tau-\tau \frac{\partial}{\partial x} x \frac{\partial}{\partial x}} \exp (-q x)\right) d r ;
$$

then after some manipulation, and using the following operational relationship [5]

$$
\exp \left(-\tau \frac{\partial}{\partial x} x \frac{\partial}{\partial x}\right) \exp (-q x)=\sum_{n=1}^{\infty} L_{n}(q x, \tau)
$$

we get the formal solution as below,

(iii) $u(x, t)=\exp \left(-\lambda t x^{v}\right) \frac{1}{\pi} \int_{0}^{\infty} \sin (\pi a \sqrt{r})\left(\int_{0}^{\infty} d \tau e^{-r \tau-\tau \frac{\partial}{\partial x} x \frac{\partial}{\partial x}} \exp (-q x)\right) d r$;

(iv) in view of relation (45)we get,

$$
u(x, t)=\exp \left(-\lambda t x^{\nu}\right) \frac{1}{\pi} \int_{0}^{\infty} \sin \left(\pi a t^{v+1} \sqrt{r}\right)\left(\int_{0}^{\infty} e^{-r \tau} \sum_{n=1}^{\infty} L_{n}(q x, \tau) d \tau\right) d r .
$$

At this point, in order to simplify the above relationship, we consider the following - well known relationship for Laguerre polynomials of two variable as below.

$$
\sum_{n=1}^{\infty} L_{n}(x, \tau)=\frac{1}{1-\tau} \exp \left(\frac{x}{1-\tau}\right)
$$


the above double integral (46) may be simplified as following

(v) $\left.u(x, t)=\exp \left(-\lambda t x^{v}\right) \frac{1}{\pi} \int_{0}^{\infty} \frac{1}{1-\tau} \exp \left(\frac{q x}{1-\tau}\right) d \tau\right)\left(\int_{0}^{\infty} e^{-\tau r} \sin (\pi a \sqrt{r}) d r\right.$; thus the result will become

$$
u(x, t)=\exp \left(-\lambda t x^{v}\right) \frac{a \sqrt{\pi}}{2} \int_{0}^{\infty} \frac{1}{\tau(1-\tau)} \exp \left(\frac{q x}{1-\tau}-\frac{\pi a^{2}}{4 \tau}\right) d \tau .
$$

\section{Main results}

Operational methods provide fast and universal mathematical tool for obtaining solution of PDEs or even FPDEs. Combination of integral transforms, operational methods and special functions give more powerful analytical instrument for solving a wide range of engineering and physical problems. This section is devoted to study exponential operators and their applications in solving certain boundary value problems, such as Laguerre heat equation of order $2 \lambda$ with non-constant coefficient.

Lemma 7.Let us consider the following space-fractional Laguerre Heat equation

$$
\begin{aligned}
& \frac{t^{-(2 k+1)}}{2(k+1)} u_{t}=\sigma\left(\frac{\partial^{\lambda}}{\partial x^{\lambda}}+(\alpha+1-x)\right)^{2} u+\delta x^{v} u(x, t), \\
& u(x, 0)=\phi(x), \quad 1.5 \leq \lambda<2 .
\end{aligned}
$$

Proof. In order to obtain a solution for equation (50) in view of [2],[3] first by solving the first order PDE with respect to $\mathrm{t}$, and applying the initial condition (51), we get the following relationship

$$
u(x, t)=\exp \left(\delta t^{2(k+1)} x^{v}\right) \exp \left(\sqrt { \sigma } t ^ { ( k + 1 ) } \left(\left(\frac{\partial^{\lambda}}{\partial x^{\lambda}}+(\alpha+1-x)\right)^{2} \phi(x),\right.\right.
$$

In order to find the result of the action of exponential operator, we can use the following well known elementary integral

$$
\exp (\eta)^{2}=\frac{1}{\sqrt{\pi}} \int_{-\infty}^{+\infty} \exp \left(-u^{2}-2 \eta u\right) d u
$$

by choosing $\eta=\sqrt{\sigma} t^{(k+1)}\left(\left(\frac{\partial^{\lambda}}{\partial x^{\lambda}}+(\alpha+1-x)\right.\right.$, to obtain

$$
u(x, t)=\exp \left(\delta t^{2(k+1)} x^{v}\right) \frac{1}{\sqrt{\pi}} \int_{-\infty}^{+\infty}(d u) \exp \left(-u^{2}-2 u\left(\sqrt{\sigma} t^{(k+1)}\left(\frac{\partial^{\lambda}}{\partial x^{\lambda}}+(\alpha+1-x)\right) \phi(x),\right.\right.
$$

after simplifying, we arrive at

$$
u(x, t)=\frac{\exp \left(\delta t^{2(k+1)} x^{v}\right)}{\sqrt{\pi}} \int_{-\infty}^{+\infty}(d u) \exp \left(-u^{2}-2 u\left(\sqrt{\sigma} t^{(k+1)}(\alpha+1-x)\right) \exp \left(-2 u \sqrt{\beta} t^{(k+1)}\left(\frac{\partial}{\partial x}\right)^{\lambda}\right) \phi(x),\right.
$$

At this point,in order to find the result of the action of exponential operator, we may use part 4 of lemma 1 by setting; $w=2 u \sqrt{\sigma} t^{(k+1)}$ and $s=\frac{\partial}{\partial x}$ to obtain

$$
\exp \left(-2 u \sqrt{\sigma} t^{(k+1)}\left(\frac{\partial}{\partial x}\right)^{\lambda}\right) \phi(x)=\frac{1}{\pi} \int_{0}^{\infty} e^{-r^{\beta}(w \cos \beta \pi)} \sin \left(w r^{\beta} \sin \beta \pi\right)\left(\int_{0}^{\infty}\left(e^{-r \tau-\tau\left(\frac{\partial}{\partial x}\right)} \phi(x)\right) d \tau\right) d r
$$


thus, it follows that

$$
\exp \left(-2 u \sqrt{\sigma} t^{(k+1)}\left(\frac{\partial}{\partial x}\right)^{\lambda}\right) \phi(x)=\frac{1}{\pi} \int_{0}^{\infty} e^{-r^{\beta}(w \cos \beta \pi)} \sin \left(w r^{\beta} \sin \beta \pi\right)\left(\int_{0}^{\infty}\left(e^{-r \tau)} \phi(x-\tau)\right) d \tau\right) d r
$$

substitution of the above result in (55),leads to the following formal solution to the space fractional Laguerre heat equation as below

$$
u(x, t)=\frac{\exp \left(\delta t^{2(k+1)} x^{\nu}\right)}{\pi \sqrt{\pi}} \int_{-\infty}^{+\infty} e^{-u^{2}-2 u \sqrt{\sigma} t^{(k+1)}(\alpha+1-x)} \int_{0}^{\infty} e^{-r^{\beta}(w \cos \beta \pi)} \sin \left(w r^{\beta} \sin \beta \pi\right)\left(\int_{0}^{\infty} e^{-r \tau} \phi(x-\tau) d \tau\right) d r d u
$$

Let us consider the special case: $\lambda=1.5, \phi(x)=\exp -(b x), \sigma=\delta=1, k=0$, we get the following heat equation

$$
\begin{array}{r}
\frac{1}{2 t} u_{t}=\left(\frac{\partial^{\frac{3}{2}}}{\partial x^{\frac{3}{2}}}+(\alpha+1-x)\right)^{2} u+x^{v} u(x, t), \\
u(x, 0)=\exp (-b x) .
\end{array}
$$

with the formal solution as below

$$
u(x, t)=\frac{\exp \left(t^{2} x^{v}\right)}{\pi \sqrt{\pi}} \int_{-\infty}^{+\infty} e^{-u^{2}-2 u t(\alpha+1-x)} \int_{0}^{\infty} \sin \left(w r^{1.5} \sin 1.5 \pi\right)\left(\int_{0}^{\infty} e^{-r \tau} \exp (-b x+b \tau) d \tau\right) d r d u
$$

if we carry out the integration and simplify the results, it follows that

$$
u(x, t)=-\frac{\exp \left(t^{2} x^{v}-b x\right)}{\pi \sqrt{\pi}} \int_{-\infty}^{+\infty} e^{-u^{2}-2 u t(\alpha+1-x)}\left(\int_{0}^{\infty} \frac{1}{r-b} \sin (2 u t r \sqrt{r}) d r\right) d u
$$

\section{Conclusion}

Operational methods provide fast and universal mathematical tool for obtaining solution of PDEs or even FPDEs. Combination of integral transforms, operational methods and special functions give more powerful analytical instrument for solving a wide range of engineering and physical problems. The paper is devoted to study exponential operators and their applications in solving certain boundary value problems. The main purpose of this work is to develop methods for solving singular integral equations, certain space fractional Lagurre heat equation. We note that within such a new frame work as we have described and developed in this article, the extensive usage of the exponential operator method opens up new and powerful possibilities, which be more deeply explored in the future publications.

\section{Acknowledgments}

The author wishes to express his sincere thanks to referee/s for valuable comments and suggestions that lead to a vast improvement in the paper.

\section{References}

[1] A.Aghili, H.Zeinali. Advances in Laplace type integral transforms with applications, Indian Journal of Science and Technology, Vol. 7(6), 877-890, June 2014.

[2] A.Aghili. New results involving Airy polynomials, fractional calculus and solution to generalized heat equation. New trends in mathematical sciences. Vol. 3, issue 4, Dec. 2015.pp 133-143. 
[3] A.Ansari. Fractional exponential operators and time fractional telegraph equation. Boundary value problems. 2012, Springer.

[4] A.Apelblat. Laplace transforms and their applications, Nova science publishers, Inc, New York, 2012.

[5] B. Davies. Integral transforms and their applications, Springer, USA, 2001.

[6] G.Dattoli. Operational methods, fractional operators and special polynomials. Applied Mathematics and computations.141 (2003) pp 151-159.

[7] G.Dattoli, H.M.Srivastava,K.V.Zhukovsky. Operational methods and differential equations to initial value problems. Applied Mathematics and computations.184 (2007) pp 979-1001.

[8] G.Dattoli, P.E.Ricci C.Cesarano.L.Vasquez. Mathematical and computer modelling.37 (2003)729-733

[9] A.A.Kilbas, H.M. Srivastava, J.J.Trujillo, Theory and applications of fractional differential equations, North Holand Mathematics Studies,204,Elsevier Science Publishers ,Amesterdam, Heidelberg and New York ,2006.

[10] I. Podlubny. Fractional Differential Equations, Academic Press, San Diego, CA,1999. 